\title{
Using integrating spheres as absorption cells: path-length distribution and application of Beer's Law
}

\author{
Jane Hodgkinson*, Dackson Masiyano and Ralph P Tatam \\ Engineering Photonics Group, School of Engineering, Cranfield University, Bedfordshire, MK43 0AL, \\ UK. \\ * corresponding author j.hodgkinson@ cranfield.ac.uk
}

\begin{abstract}
We have modeled the path-length distribution in an integrating sphere used as a multipass optical cell for absorption measurements. The measured radiant flux as a function of analyte concentration is nonlinear as a result, deviating from that expected for a single path length. We have developed a full numerical model and introduce a new analytical relationship that describes this behavior for high reflectivity spheres. We have tested both models by measuring the optical absorption of methane at $1651 \mathrm{~nm}$ in a $50 \mathrm{~mm}$ diameter sphere, with good agreement with experimental data in the absorption range $0-0.01 \mathrm{~cm}^{-1}$. Our results compare well with previous work on the temporal response of integrating spheres.
\end{abstract}

\section{OCIS codes}

$120.3150 \quad$ Integrating spheres

$120.6200 \quad$ Spectrometers and spectroscopic instrumentation

300.1030 Absorption

280.4788 Optical sensing and sensors 


\section{Introduction}

Interest is growing in the use of integrating spheres as optical absorption cells. Light entering the sphere is scattered by the internal walls with very high levels of total diffuse reflectance. For an ideal diffuse scatterer or Lambertian surface, the radiance $L$ (in $\mathrm{W} \mathrm{m}^{-2} \mathrm{sr}^{-1}$ ) from a given point is a constant in any direction. For a cavity with spherical geometry, it follows that the irradiance $E$ (in $\mathrm{W} \mathrm{m}^{-2}$ ) received from that point is then constant over the entire sphere ${ }^{[1,2]}$. Therefore, after only a few passes across the cell to remove the local effects of launch geometry, the irradiance is perfectly uniform over the surface. This makes integrating spheres ideal for use in measurement of parameters such as the total emission from light sources.

For the measurement of absorption, advantages include a tolerance to misalignment and the ability to measure irregular or turbid samples without the scattered light affecting the measurement. In 1970, Elterman $^{[2]}$ demonstrated measurements of glass samples at $546 \mathrm{~nm}$ within a simple $3.2 \mathrm{~cm}$ diameter integrating sphere. Fry, Kattawar and Pope ${ }^{[3]}$ have measured visible absorption in aqueous solutions, Fecht and Johnson ${ }^{[4]}$ have adapted the principle for use with falling aqueous sample streams, and Hodgkinson $e t$ $a l$. have adapted a photothermal cell using an integrating cavity ${ }^{[5]}$.

To our knowledge, the use of an integrating sphere for gas absorption measurements was first reported in 1980 by Venkatesh et al ${ }^{[6]}$ for carbon monoxide monitoring around $5 \mu \mathrm{m}$. In 1988 Abdullin and Lebedev ${ }^{[7]}$ used a copper sphere with a $\mathrm{CO}_{2}$ laser to measure ammonia concentrations and in 1996 Tranchart et al. ${ }^{[8]}$ used a $10 \mathrm{~cm}$ diameter sphere in combination with tunable diode laser spectroscopy at $830 \mathrm{~nm}$ and $1.2 \mu \mathrm{m}$ to detect water vapor and butane respectively. These studies confirmed an important advantage of using integrating spheres. Compared with standard multipass optical cells such as those developed by White ${ }^{[9]}$, 
Herriott et al. ${ }^{[10]}$ or Chernin et al. ${ }^{[11]}$, integrating spheres offer increased path lengths within a relatively small volume without the need for precise alignment of the laser beam.

More recent work at the University of Limerick has resulted in broadband spectroscopic sensors for $\mathrm{CO}_{2}$ (at $1.57 \mu \mathrm{m}$ and $2 \mu \mathrm{m})^{[12]}$ and $\mathrm{NO}_{2}$ and $\mathrm{SO}_{2}$ (in the UV/visible) ${ }^{[13]}$. Finally, a commercial non-dispersive infrared (NDIR) gas sensor designed by City Technology Ltd. takes the form of an integrating cavity, using deliberately roughened, gold coated internal surfaces ${ }^{[14]}$.

At low concentrations, the level of light absorption measured in such systems is proportional to the concentration of the analyte. However, many practical systems are required to operate over a large dynamic range that might extend into a region of nonlinearity for absorption measurements. In particular, the lower explosive limit (LEL) for methane is 4.9 vol. $\%^{[15]}$, defining a concentration range in \% LEL where $100 \%$ LEL corresponds to 4.9 vol. \% It can be important for methane detectors in safety critical applications to be able to cover the entire range from low ppm levels up to and through the \%LEL range, with particular attention paid to measurements at $20 \%$ LEL (approx 1 vol. \% methane) ${ }^{[15]}$.

For a gas cell with a single path length, the Beer-Lambert Law (see Section 2) governs nonlinearity in the measured light intensity as a function of analyte concentration. However, for a cell such as an integrating sphere where the light makes multiple randomized passes with different path lengths, the nonlinearity is exacerbated and dependent on the path-length distribution within the cavity. Previous work in this area has so far been concerned with calculating the average path length of photons in the cavity ${ }^{[16,17]}$, or calculating the decay time of a pulse of photons ${ }^{[17]}$ using a Monte Carlo simulation, the latter also providing more general information about the distances travelled by photons in the cavity.

We have taken this work a stage further by modeling the path-length distribution within an integrating 
sphere and predicting the resulting system nonlinearity. We have compared this prediction with a simple experimental absorption measurement based on tunable diode laser spectroscopy of a single methane line at $1651 \mathrm{~nm}$. This provides an experimental test not only for our predicted absorption response, but also for previous work on temporal responses.

\section{Theory}

Figure 1 shows a simplified model of the integrating sphere used in our experiments. For monochromatic radiation, the radiant flux (in watts) transmitted through a gas cell, $\Phi$, is given by the Beer-Lambert law ${ }^{[18]}$ :

$$
\Phi=\Phi_{0} \exp (-\alpha z)
$$

Where $\Phi_{0}$ is the radiant flux transmitted in the absence of light absorption, $z$ is the path length (here in centimeters) and $\alpha$ is the absorption coefficient of the sample, equal to the concentration of the analyte (here expressed in units of partial pressure, atmospheres, equivalent to volume \% readings at atmospheric pressure), multiplied by the specific absorptivity of the gas $\varepsilon$ (here in units atm ${ }^{-1} \mathrm{~cm}^{-1}$ ). Methane has a wellknown absorption line at a wavelength of $1651 \mathrm{~nm}$. At atmospheric pressure the value of $\varepsilon$ at the line center is $0.38 \mathrm{~cm}^{-1} \mathrm{~atm}^{-1}$, according to information in the HITRAN database ${ }^{[19]}$. 


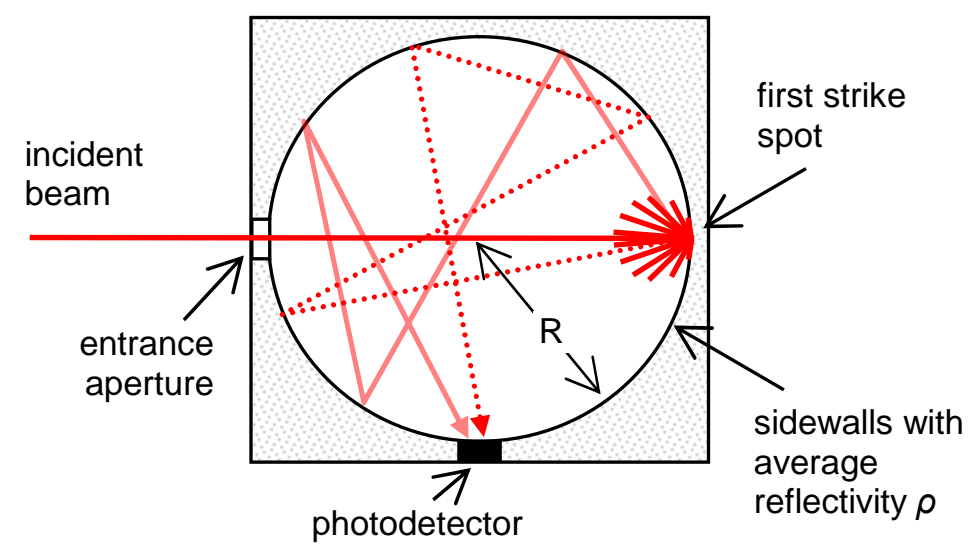

Figure 1. Simplified model of an integrating sphere, showing the incident beam making a first pass across the cell to the first strike spot. Two examples are shown of light beams making subsequent random multiple passes across the cell.

At low values of $\alpha z$, Eq. (1) approximates to the following linear relationship.

$$
\frac{\Delta \Phi}{\Phi_{0}} \approx \alpha z
$$

Where $\Delta \Phi$ is the change in transmitted radiant flux, $\Phi_{0^{-}} \Phi$. Tranchart et al. ${ }^{[8]}$ have shown that for an integrating sphere of internal radius $R$ and high values of mean surface reflectivity $\rho$, the received signal for low $\alpha z$ is approximated by;

$$
\frac{\Delta \Phi}{\Phi_{0}} \approx \frac{\alpha z_{0}}{(1-\rho)}
$$

where the mean path length for a single pass is $z_{0}=\frac{4}{3} R$. They then derive the effective total path length for low or zero absorbance $\alpha$ within the sphere, $Z_{\text {eff; }}$;

$$
Z_{\text {eff }} \approx \frac{4}{3} \frac{R}{(1-\rho)}
$$

In the next section we derive equations that describe the performance of the sphere over the full range of gas concentrations to give a more complete description of sphere performance. 


\section{A Simple model for path-length distribution}

For gas cells involving a range of different path lengths, it is necessary to consider the contribution that each individual path length makes separately, before integrating over all path lengths. We define the probability density function (PDF) $\Psi(z)$ of path lengths to give the proportion of path lengths that lie within the interval $z$ to $z+\mathrm{d} z$, in the absence of gas, such that

$$
\int_{0}^{\infty} \Psi(z) d z=1
$$

Note that for this model, we have normalized $\Psi(z)$ to the total level of light received at the detector in the absence of gas, rather than the total incident into the sphere. We apply the Beer-Lambert Law (Eq. (1)) to each path length in the interval from $\mathrm{z}$ to $\mathrm{z}+\mathrm{dz}$, such that the radiant flux at the detector at a given wavelength is then;

$$
\Phi=\Phi_{0} \int_{0}^{\infty} \Psi(z) \exp (-\alpha z) d z
$$

A simple expression can be derived for integrating spheres with high internal reflectivities, in which the path-length distribution for a single pass across the cell is neglected and each pass is considered to have length equal to the mean path length of $\mathrm{z}_{0}=\frac{4}{3} \mathrm{R}$. For such a cell, Fry et al. ${ }^{[17]}$ have derived the temporal response of the irradiance incident on the walls, $E\left(\mathrm{in} \mathrm{W} \mathrm{m}^{-2}\right)$, following an impulse. The irradiance as a function of time, $\mathrm{E}(\mathrm{t})$, is given by;

$$
E(t)=E_{0} \exp \left(-\frac{t}{\tau}\right) \quad \text { and } \quad \tau=\frac{-\bar{t}}{\ln \rho}
$$

Where $\bar{t}$ is the mean transit time for photons across the cavity. We can transform from time to distance as

a variable for the impulse, using $\mathrm{z}=\mathrm{ct}$ where $\mathrm{c}$ is the speed of light in the cavity; we also have $z_{0}=c \bar{t}$. The irradiance resulting from photons with a transit distance $\mathrm{z}, \mathrm{E}(\mathrm{z})$, is therefore 


$$
E(z)=E_{0} \exp \left(-\frac{z}{\delta}\right) \quad \text { and } \quad \delta=\frac{-z_{0}}{\ln \rho}
$$

For a fixed detector and continuous wave $(\mathrm{CW})$ input, this also, therefore, gives the $\mathrm{z}$ dependency for the fractional radiant flux; for simplicity we separate out this z dependency as a normalized probability density function for optical path lengths, such that;

$$
\Psi(z)=\Psi_{0} \exp \left(-\frac{z}{z_{0}} \ln \rho\right)
$$

where $\Psi_{0}$ is a normalization constant. Substitution into Eq. (6) and normalizing gives

$$
\frac{\Phi}{\Phi_{0}}=\frac{\int_{0}^{\infty} \exp \left(\frac{z}{z_{0}} \ln \rho-\alpha z\right) d z}{\int_{0}^{\infty} \exp \left(\frac{z}{z_{0}} \ln \rho\right) d z}
$$

which evaluates to the general expression

$$
\frac{\Phi}{\Phi_{0}}=\frac{\ln \rho}{\ln \rho-\alpha z_{0}} .
$$

For low $\alpha z_{0}$ and high $\rho$, this approximates to Eq. (3) in this paper, taken from Tranchart et al. ${ }^{[8]}$.

It was necessary to account for specific launch and de-launch conditions as follows. Our first pass across the sphere took the form of a collimated beam from the entry point to the first strike spot, with a path length of $2 \mathrm{R}$. In our experimental integrating sphere, any final pass would involve a detector with a field of view restricted to $\pm 30^{\circ}$. Figure 2 and Table 1 summarize consecutive passes across the cell, indicating how each pass was modeled. Eq. (10) fails to take account of the first and last pass across the cell; to do so, we multiplied by the transmission expected from the Beer-Lambert law for the additional launch / delaunch path, $z_{\ell}$, estimated in Table 1 to be $2.52 \mathrm{R}$. We also add an additional offset path length of $z_{0} / 2$ to account for averaging effects over the first few passes (see later in Figure 9(b) for a graphical explanation). The offset ensures that both our analytical and our full numerical models converge to the same mean path length after approximately 3-4 passes across the cell, giving the following combined equation: 


$$
\frac{I}{I_{0}}=\exp \left(-\alpha\left(z_{\ell}+\frac{z_{0}}{2}\right)\right) \frac{\ln \rho}{\ln \rho-\alpha z_{0}}
$$

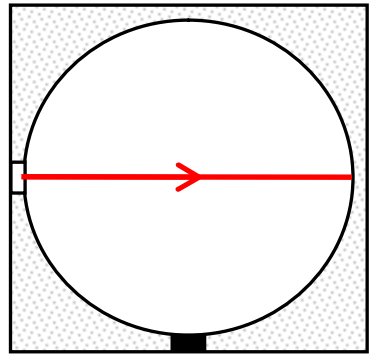

(a) First pass: pathlength $z_{1}=2 R$

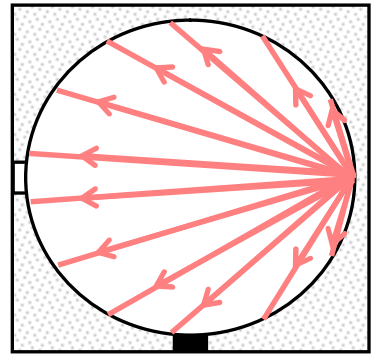

(b) Second (and subsequent) passes: mean pathlength

$$
z_{0}=\frac{4}{3} R
$$

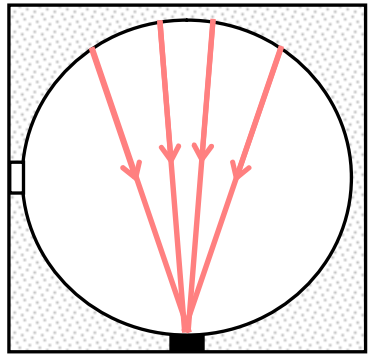

(c) Final pass for detector with restricted field of view: mean pathlength $1.85 R$

Figure 2. Illustration of different phases of a photon's journey within the integrating sphere, from entrance aperture to detector 
Table 1. Summary of modelled passes across the integrating sphere.

\begin{tabular}{|l|l|c|c|l|}
\hline$\underline{\text { Pass }}$ & Target & $\underline{\text { Reflectivity of target }}$ & $\underline{\text { Mean path length }}$ & $\underline{\text { Nature of model }}$ \\
\hline 1 & First strike spot & $1^{\mathrm{a}}$ & $2 \mathrm{R}$ & Launch condition \\
\hline 2 & Inner surface & $\rho$ & $\frac{4}{3} \mathrm{R}$ & Eq. (12) and full numerical model \\
\hline$\vdots$ & $\vdots$ & $\vdots$ & $\vdots$ & $\vdots$ \\
\hline $\mathrm{n}-1$ & Inner surface & $\rho$ & $\frac{4}{3} \mathrm{R}$ & Eq. (12) and full numerical model \\
\hline $\mathrm{n}$ & Detector & $\rho^{\mathrm{b}}$ & $\frac{4}{3} \mathrm{R}+0.52 \mathrm{R}^{\mathrm{c}}$ & $\begin{array}{l}\text { Both models plus de-launch } \\
\text { condition }\end{array}$ \\
\hline
\end{tabular}

a Light cannot reach the detector immediately after this pass, therefore normalisation removes the effect of non-unity reflectivity and we model it as $\rho=1$.

b We assume that the detector simply samples light representative of any point on the surface, therefore make no adjustment for a specific detector at a specific location.

c Our detector had a restricted view of $\pm 32^{\circ}$. Using Eq. (13), the mean path length for any final pass to the detector evaluates to $1.85 \mathrm{R}$; an additional $0.52 \mathrm{R}$.

\section{B Full model for path-length distribution}

A full model requires calculation of the path-length distribution for a single pass across the cell from a Lambertian scatterer. The geometry of this pass is illustrated in Figure 3 and has been considered by Tranchart et al. ${ }^{[8]}$; for clarity we have continued to use their formalism. 


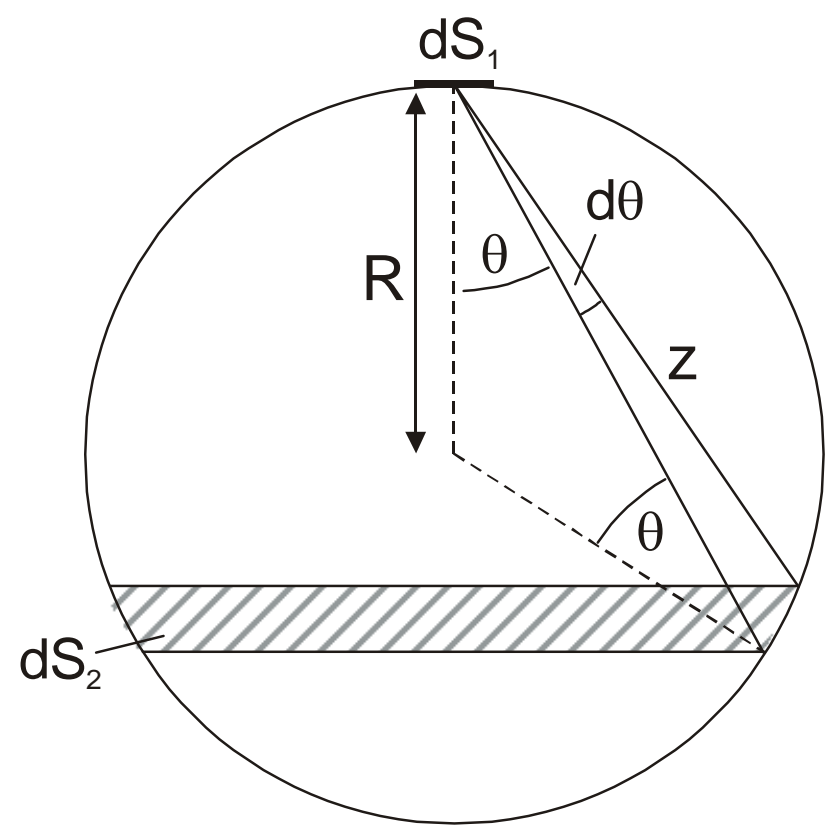

Figure 3. Geometry used to model integrating sphere path-length distribution, including elemental surface areas $\mathrm{dS}_{1}$ and $\mathrm{dS}_{2}$.

Tranchart et al. have shown the radiant flux $\Phi$ in Watts emitted by the annulus $\mathrm{d} S_{2}$ towards the area $\mathrm{d} S_{l}$ is given by

$$
\frac{\mathrm{d}^{2} \Phi}{\mathrm{d} S_{1}}=\frac{L \cos ^{2} \theta}{z^{2}} \mathrm{~d} S_{2}
$$

where $L$ is the radiance of the surface of the sphere (in units of $\mathrm{W} \mathrm{m}^{-2} \mathrm{sr}^{-1}$ ), which can be considered constant for an ideal sphere. Now $\cos \theta=z / 2 R$, giving

$$
\frac{\mathrm{d}^{2} \Phi}{\mathrm{d} S_{1}}=\frac{L}{4 R^{2}} \mathrm{~d} S_{2}
$$

Since $d S_{2}=2 \pi z d z$

$$
\frac{\mathrm{d}^{2} \Phi}{\mathrm{d} S_{1}}=\frac{\pi L z}{2 R^{2}} \mathrm{~d} z
$$


We normalize to the total radiant flux received by $\mathrm{d} S_{1}$, setting the value of $\Phi$ as follows;

$$
\mathrm{d} \Phi=\pi L \mathrm{~d} S_{1}
$$

The PDF of path lengths for a single pass across the cell, $\psi$, is defined as the proportion of light received at $\mathrm{d} S_{l}$ within an interval from $z$ to $z+\mathrm{d} z$ :

$$
\psi(z)=\frac{\mathrm{d} \Phi}{\mathrm{d} z}=\frac{z}{2 R^{2}}
$$

As a check, we can calculate the mean path length across the sphere as follows;

$$
z_{0}=\int_{0}^{2 \mathrm{R}} z \psi(z) \mathrm{d} z
$$

which evaluates to $z_{0}=\frac{4}{3} \mathrm{R}$, as expected.

Now consider multiple passes across the cell, as illustrated in Figure 1. Because light scattering is completely randomized, each pass is independent of the next. From one pass to the next, the total irradiance is reduced in proportion to $\rho$. Importantly, for a Lambertian scatterer the diffuse reflectivity is not a function of incident angle, therefore within a sphere all light paths in one pass are equally likely to create light paths of the same distribution in a subsequent pass.

After the first pass, the path-length distribution is equal to $\psi(z)$. A proportion of light paths $(1-\rho)$ do not survive to the second pass, whereas a proportion of paths $\rho$ go on to make a second pass. After the second pass, each surviving path in the interval $z+\mathrm{d} z$ is added cumulatively to further path lengths in proportion to the path-length distribution $\psi(z)$, to give a distribution $\Psi(z)$. And so on.

This reasoning does not lend itself to an analytical solution, however it has been possible to build a numerical model to give a full path-length distribution (in the absence of gas) for multiple passes. We have considered the full model alongside the simpler model of Eq. (10), in which the path-length distribution for a single pass is taken to be equal to a constant value equal to $\frac{4}{3} R$. Comparing these models therefore allows 
us to evaluate whether the single-pass distribution makes a significant contribution, or whether each pass across the sphere can be adequately modeled using the mean path length of $\frac{4}{3} R$.

\section{Numerical modeling of path lengths}

Our numerical model is illustrated in Figure 4. We established a discrete series of $\mathrm{N}$ path-length intervals per sphere radius, with $\mathrm{N}=100$ giving a resolution equal to $\mathrm{R} / 100$. As a total of 500 passes were modeled, the maximum theoretical path length that could be achieved was $1000 \mathrm{R}$, split into $100 \times 500=50,000$ discrete intervals. For the first pass, the values of path lengths $z_{j}$ and the corresponding probability density function (PDF) $\psi_{j}$ were given by Eq. (17). Let these be assigned to an overall cumulative distribution of path lengths $Z_{i}$ with probabilities given by $\Psi_{i}$.

For the second pass, each of the $Z_{i}$ paths from the first pass could add to any of the paths $z_{j}$ from the second pass. The corresponding probability is given by $\Psi_{i \cdot} \psi_{j} . \rho$. This procedure yielded two matrices corresponding to all possible summed path lengths and their corresponding probabilities. A re-binning exercise created new cumulative vectors $Z_{i}$ (by sorting and summing the path-length matrix) and $\Psi_{i}$ (by following the mapping for the path lengths $Z_{i}$ and summing probabilities). 


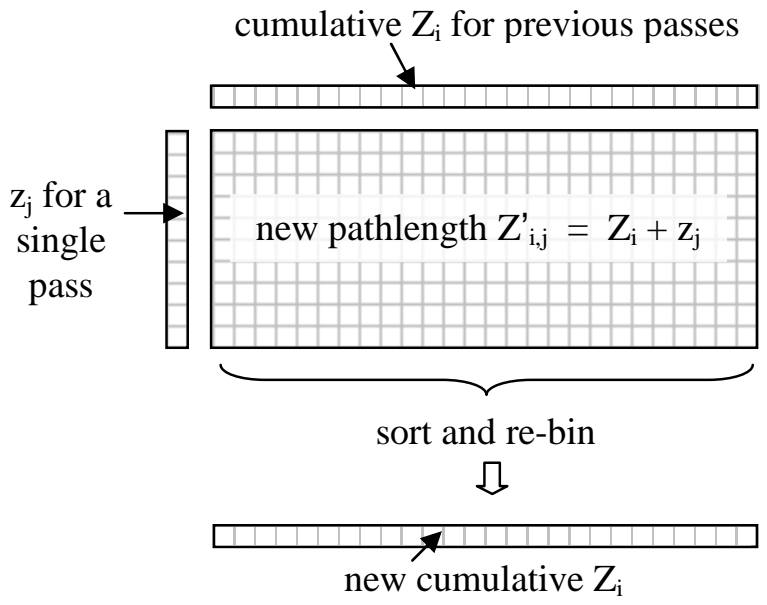

(a) Modelling procedure for pathlengths

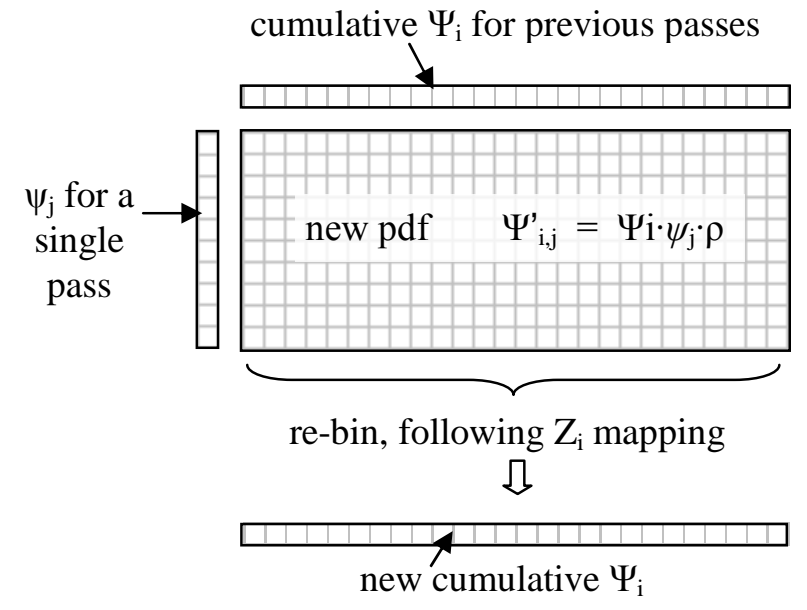

(b) Modelling procedure for PDF

Figure 4. Illustration of numerical modeling procedure for path-length distribution, (a) determining the values of path lengths in discrete intervals, and (b) determining the corresponding PDF.

There was the potential to introduce a rounding error during the re-binning exercise because of the finite resolution of the bin size. For each modeled pass a single rounding error for one bin would be equal to half the width of one interval, or R/N. If randomly distributed, over 500 passes these could combine to a total error for the final cumulative path length $Z_{i}$ in each bin of $\sqrt{500} \mathrm{R} / \mathrm{N}=0.2 \mathrm{R}$.

Note that so far, this model has accounted only for those photons that survived 500 successive passes across the cell. We accounted for those that were absorbed at the sphere surface as follows. For each iteration of a pass across the sphere, a proportion $\rho$ of all the light survived to the next pass and a proportion $(1-\rho)$ was absorbed. A second cumulative matrix was constructed, corresponding to the photons that did not survive. Over the course of 500 iterations, the majority of the modeled light accumulated within this second matrix; indeed, has it not done so, it would have been necessary to extend the number of iterations to ensure that the model converged to a solution. Had we not considered the photons that did not survive, the model would have predicted the unphysical case of a vanishingly small amount of light that persisted in a cell with 
a path length approaching infinity.

We normalized to the total level of light that reached the detector, rather than the total incident at the sphere

entry port, therefore requiring that $\sum \Psi_{i}=1$. Because the role of the detector as an absorber was already accounted for in the value of the absorbed fraction of light, (1- $\rho)$, we did not specifically account for its size. Finally we accounted for launch / delaunch geometry by adding an additional distance of $z_{\ell}=2.52 \mathrm{R}$ to all the modeled paths, following Figure 4 and Table 1.

Our results took the form of a vector of discrete path-length intervals $Z_{i}$, alongside a corresponding vector containing the fraction of light $\Psi_{i}$ that was modeled to lie within the interval $Z_{i}+\delta z$. To predict the fraction of light that would then be received by a detector in the presence of a gaseous absorber filling the sphere, Eq. (6) was evaluated using the discrete values of $Z_{i}$ and $\Psi_{i}$.

\section{Experimental details}

To test our models, we chose to use a simple form of direct spectroscopy, in which the emission from a tunable diode laser was scanned across a methane line. This had the advantage of simplicity of operation and made a direct measurement of the depth of an absorption line whose peak absorption is well-known. Our experimental arrangement is shown schematically in Figure 5. 


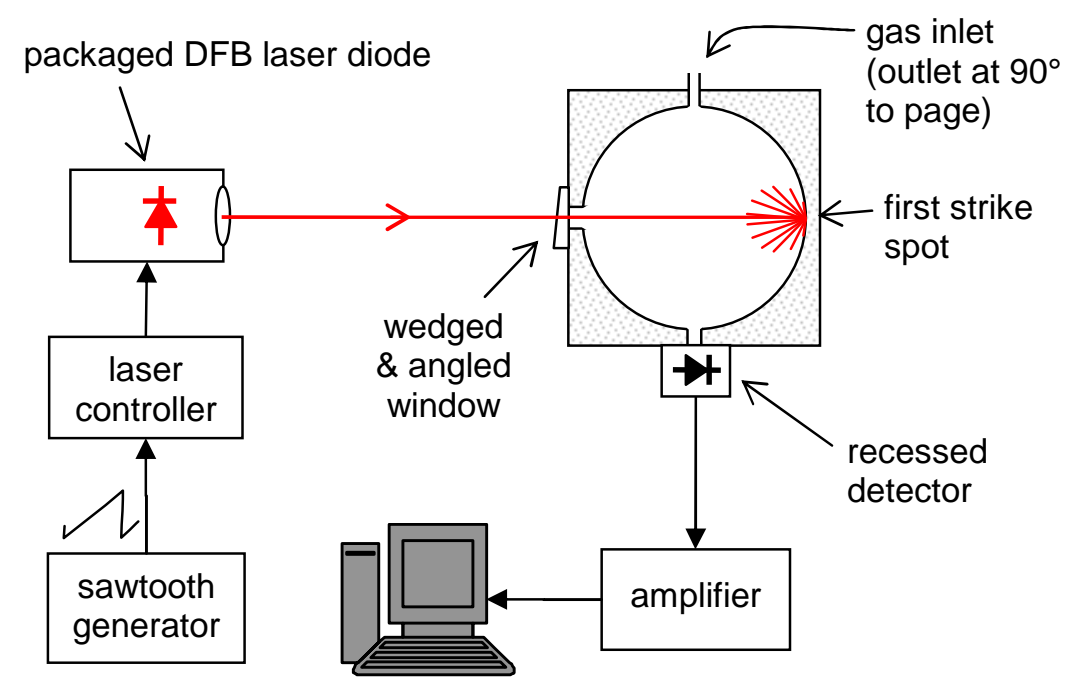

Figure 5. Experimental configuration used for direct line scanning or wavelength modulation spectroscopy of methane absorption in an integrating sphere.

Our DFB laser package (Semelab Ltd) incorporated a $1651 \mathrm{~nm}$ laser diode (NEL NLK1U5C1CA-TS). The emitted wavelength was scanned across the gas absorption line center by applying a current ramp through a driver (ILX Lightwave, LDC-3722B), the current varying between a minimum of 30mA and a maximum of $80 \mathrm{~mA}$ (corresponding to a frequency range of $33 \mathrm{GHz}$ ). Gross wavelength tuning was achieved by controlling the diode temperature using a Peltier element within the package. The wavelength of the emission was checked, relative to the methane absorption line, using a gas reference cell consisting of a Ge detector in a TO can filled with $100 \%$ methane (Semelab Ltd) to give a reference path length of approximately $5.3 \mathrm{~mm}$.

A detector / amplifier (Thorlabs PDA400) was used with a gain of $0 \mathrm{~dB}(15 \mathrm{kV} / \mathrm{A})$. The output was sampled using a data acquisition card and transferred to a PC for data processing. In the absence of gas, a reference measurement showed a rising output intensity with current, as expected. In the presence of methane, at the absorption line centre a depression was observed corresponding to the scale of the absorption (Figure 6(a)). 
The peak absorption was measured by subtracting the received signal from the reference and normalizing to the reference intensity (Figure 6(b)).

An integrating sphere was chosen with a diameter of $50.8 \mathrm{~mm}$ (Thorlabs IS200). Light from the laser diode entered the integrating sphere via a $6.35 \mathrm{~mm}$ diameter entry port. The detector $(12.5 \mathrm{~mm}$ diameter port) was recessed to prevent a direct line of sight light from the first strike spot; therefore, no baffle was necessary. The detector's field of view was restricted to $\pm 32^{\circ}$. Two additional ports $(1.5 \mathrm{~mm}$ diameter) were modified so as to permit entry and exit of test gas concentrations.

Test gases were fed to the sphere from two certified cylinders (Scott Specialty Gases), one containing hydrocarbon (HC) free air and the other containing 2.5 vol.\% methane in air. Two calibrated mass flow controllers (Teledyne Hastings HFC-302, 0-1000 cm³/min, with THPS-400 controller) were used to control flow rates from the two cylinders, with downstream mixing generating a series of mixtures of different concentrations in the range $0-2.5 \mathrm{vol} . \%$, corresponding to an absorption range of $\alpha=0-0.01 \mathrm{~cm}^{-1}$. The error in the applied concentration was dominated by the analytical error in the gas cylinder concentration $( \pm 1 \%$ of the nominal value), except at low flow rates through the mass flow controllers, where an additional error of $0.5 \%$ of the full scale reading applied in the worst case.

\section{Results}

Figure 6 (a) shows an example of raw data collected directly from the detector, for $\mathrm{HC}$ free air and for a mid range methane concentration of 5770ppm. Figure 6 (b) shows the results following processing to subtract the zero baseline from the signal and normalize for the total received light. The drop in transmission at the absorption line centre is apparent in the figure, and this was used as our measurement. The experimental error in the transmission for each measurement was estimated to be $\pm 0.3 \%$. Using a 
single measurement of direct absorption had the disadvantage of a relatively high level of experimental noise at low concentrations; however, this method offered accurate measurement of the transmission, without systematic errors.
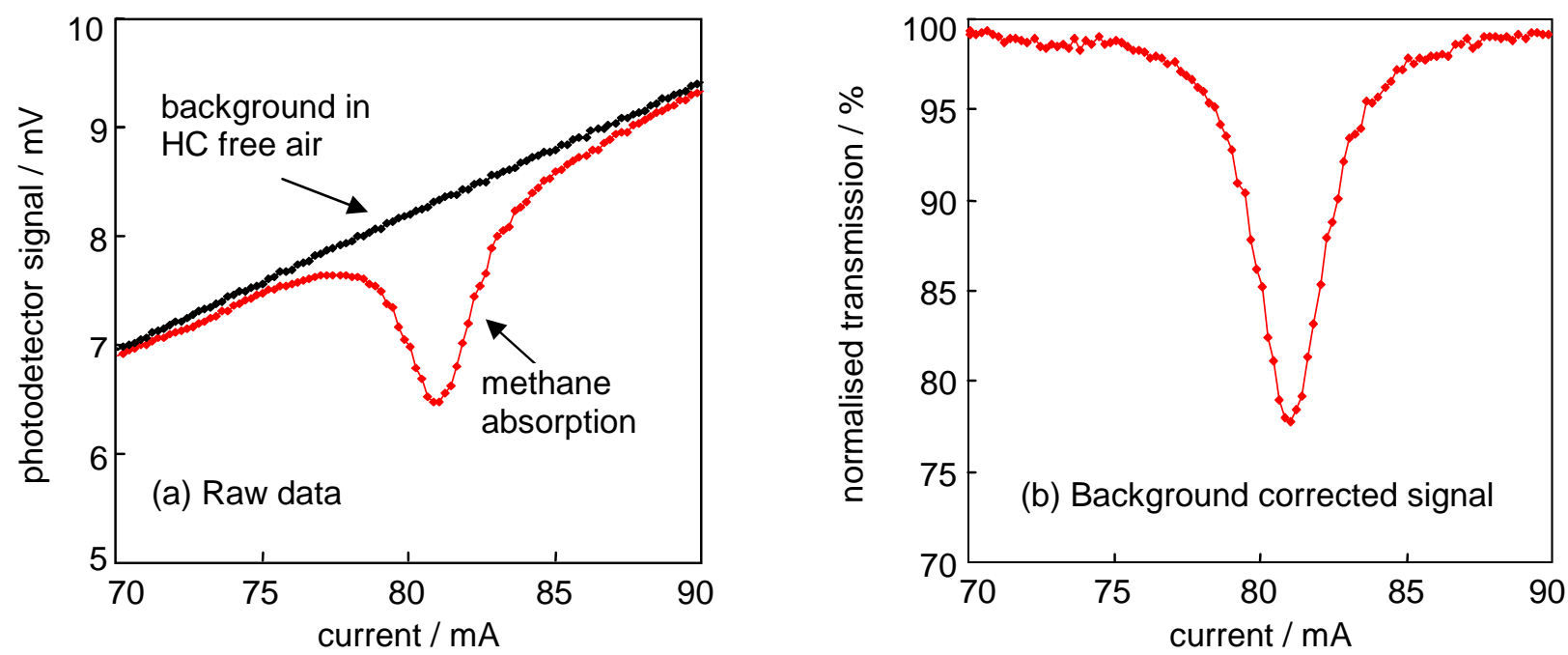

Figure 6. Examples of detected signals recorded while the laser wavelength was scanned across a single methane line at $1651 \mathrm{~nm}$. (a) Raw data from the detector for $\mathrm{HC}$ free air and for a methane concentration of $5770 \mathrm{ppm}$. (b) Methane transmission normalized by division by the background signal.

\section{A Calibrating the model}

Eq. (4) confirms that the average reflectivity $\rho$ of the inner surface of an integrating sphere has a strong influence on the mean effective path length. Manufacturer's data provides an approximate value for $\rho$, but measurement difficulties prevent a more precise value being known for a given sphere. Furthermore, light sinks such as entry ports, ports for gas sampling and the presence of a detector reduce the effective reflectivity $\rho$ of the sphere as a whole. It was therefore necessary to calibrate the value of $\rho$ used in our model with some initial experimental results. 
We calibrated the sphere at our lowest experimental concentrations of $0 \mathrm{ppm}$ and $2270 \mathrm{ppm}$, with transmission at the line center of $100 \%$ and $96.51 \pm 0.14 \%$, respectively. Eq. (1) was used to fit these results to a mean path length of $Z_{\text {eff }}=1.22 \pm 0.04 \mathrm{~m}$. To account for launch geometry with $\mathrm{z}_{\ell}=2.52 \mathrm{R}$, we used the value of $Z_{\text {eff }}-z_{\ell}=1.16 \pm 0.04 \mathrm{~m}$ for calibration. Eq. (4) (which does not provide for these launch conditions) was then used to find the average sphere reflectivity of $\rho=97.11 \pm 0.10 \%$.

\section{B Testing the simple analytical model}

We tested the model of Eq. (12), which assumes that for each pass across the cell, the path length takes a

single value equal to $z_{0}=\frac{4}{3} \mathrm{R}$. The model was tested using our calibration value of $\rho=97.11 \%$ and launch / delaunch path length $z_{\ell}=2.52 \mathrm{R}$. We were also able to fit these parameters to our data to optimise this model, resulting in optimal values of $\rho=97.13 \%$ and launch / delaunch path length $z_{\ell}=2.85$ R. Figure 7 compares our experimental data with the modeled results, plus the prediction of the Beer Lambert law for a single path-length cell. The graph illustrates the scale of the deviation from a simple application of this law, and the need for a better model. The results imply that additional care needs to be taken for gas measurement schemes that use direct absorption scans with a fitted line shape, rather than with simple measurement of the transmission at the line centre. Such schemes would be vulnerable to apparent changes in the line shape caused by transmission nonlinearities. 


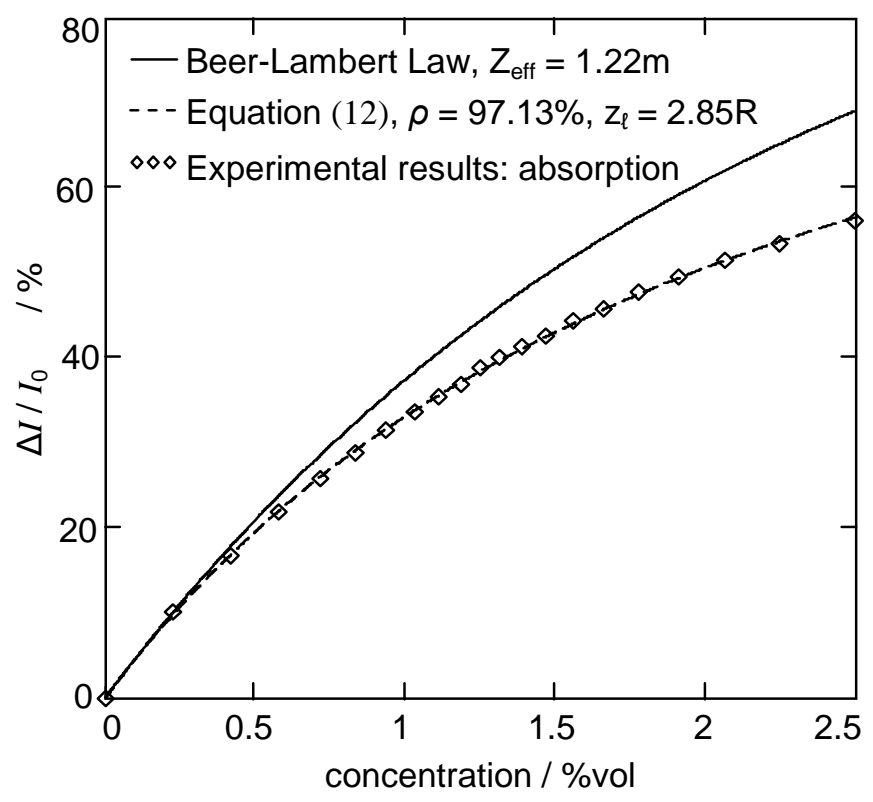

Figure 7. Comparison between experimentally determined response and the results of Eq. (12) using optimized values of $\rho$ and $z_{\ell}$. Also shown for comparison is the response predicted by the Beer Lambert law for a single path-length cell.

Figure 8 shows the residual errors in the modeled response, compared to our experimental data. Our total experimental error results from the combined effects of a $0.3 \%$ error in our transmission reading and the error in the gas concentration. For the purpose of comparison, the latter has been transformed to an equivalent transmission error using Eq. (12) and combined with the former to yield a total effective error of between $0.4 \%$ and $0.5 \%$ on the transmission data.

Visual inspection reveals that the agreement between the simplified model and our experimental data is within our estimated experimental error. The residual errors in Figure 8 show a consistent pattern as a function of concentration. This may be associated with an underlying error in our experimental data, which would persist in comparisons with different models. A small step change is visible between readings at 
concentrations around $1.2 \%$ vol, but it is not associated with any obvious experimental factor such as a change of mass flow controller or any other change.

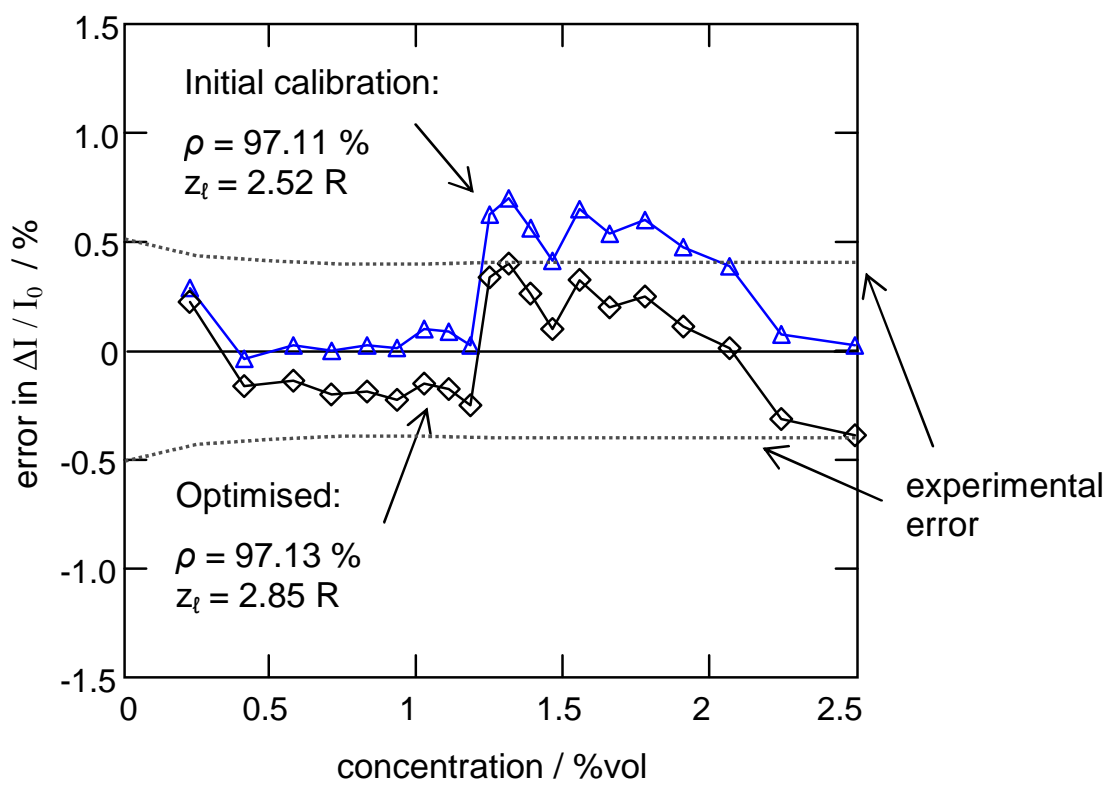

Figure 8. Residual error in modeled response of the system (as $\Delta \mathrm{I} / \mathrm{I}_{0}$ ) compared to experimental results, comparing the results of Eq. (12) for different values of $\rho$ and $z_{\ell}$. Agreement with the optimized model is within our estimated experimental error.

\section{Numerically modeled path-length distributions}

We chose to complete a sensitivity analysis by modeling spheres with reflectivities covering the range $\rho=96.7 \%$ to $97.5 \%$ in steps of $0.1 \%$. Examples of three probability density functions (PDFs) are plotted in Figure 9, showing the distribution of light in different path-length intervals. These PDFs shows the results of our full model that takes into account the distribution of path lengths for a single pass across the sphere. Note that the $\mathrm{x}$-axis is logarithmic and the PDFs are normalized such that the integrated area under each 
curve equals unity. As $\rho$ increases, there is a shift in the path-length distribution toward longer path lengths, as expected. At short path lengths, the effect of consecutive passes across the sphere can be distinguished as a series of undulations in the PDF, but with increasing passes across the sphere these merge into one another until they can no longer be resolved. The PDF then converges to the exponentially decaying function predicted by Eq. (9).

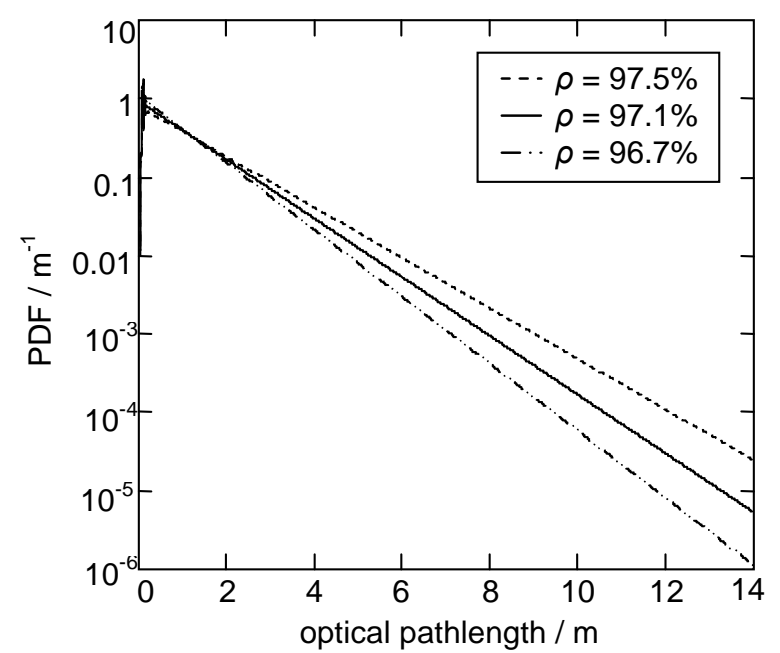

(a)

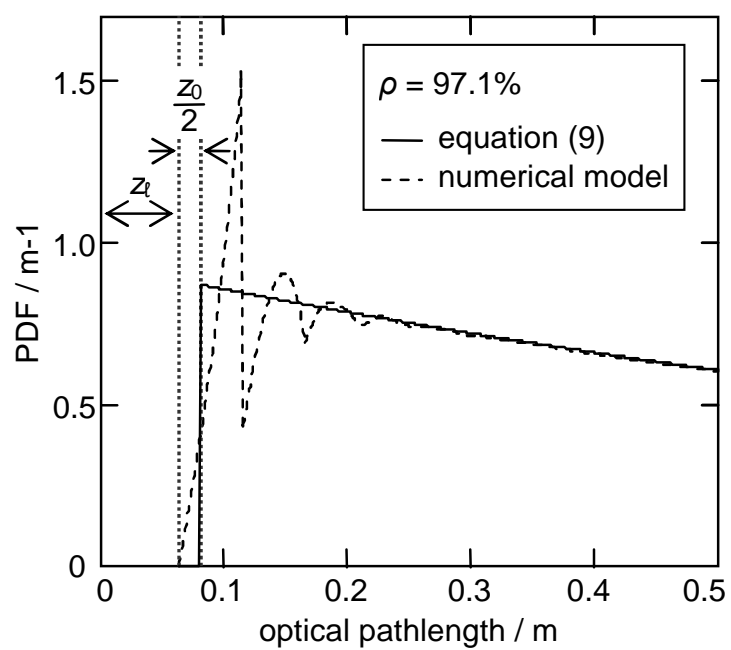

(b)

Figure 9. Optical path-length distribution for a $50.8 \mathrm{~mm}$ diameter sphere. (a) Numerical model for three different average sphere reflectivities, (b) expanded linear scale showing the correspondence between the numerical and analytical models for the first few passes across the cell. The launch path $z_{\ell}$ and the offset $z_{0} / 2$ (see Eq. (12)) are indicated for clarity.

\section{Testing the full numerical model}

The modeled path-length distributions were used to predict the level of light absorbed by the $1651 \mathrm{~nm}$ methane line at the line center, using Eq. (6). The results for a range of surface reflectivities $\rho$ are compared 
with our experimental data in Figure 10 and show good agreement. For the purpose of comparison, the prediction given by Eq. (1) (the Beer-Lambert law) is given using the experimentally determined mean path length of the sphere in the absence of absorption, $\mathrm{Z}_{\mathrm{eff}}=1.22 \mathrm{~m}$.

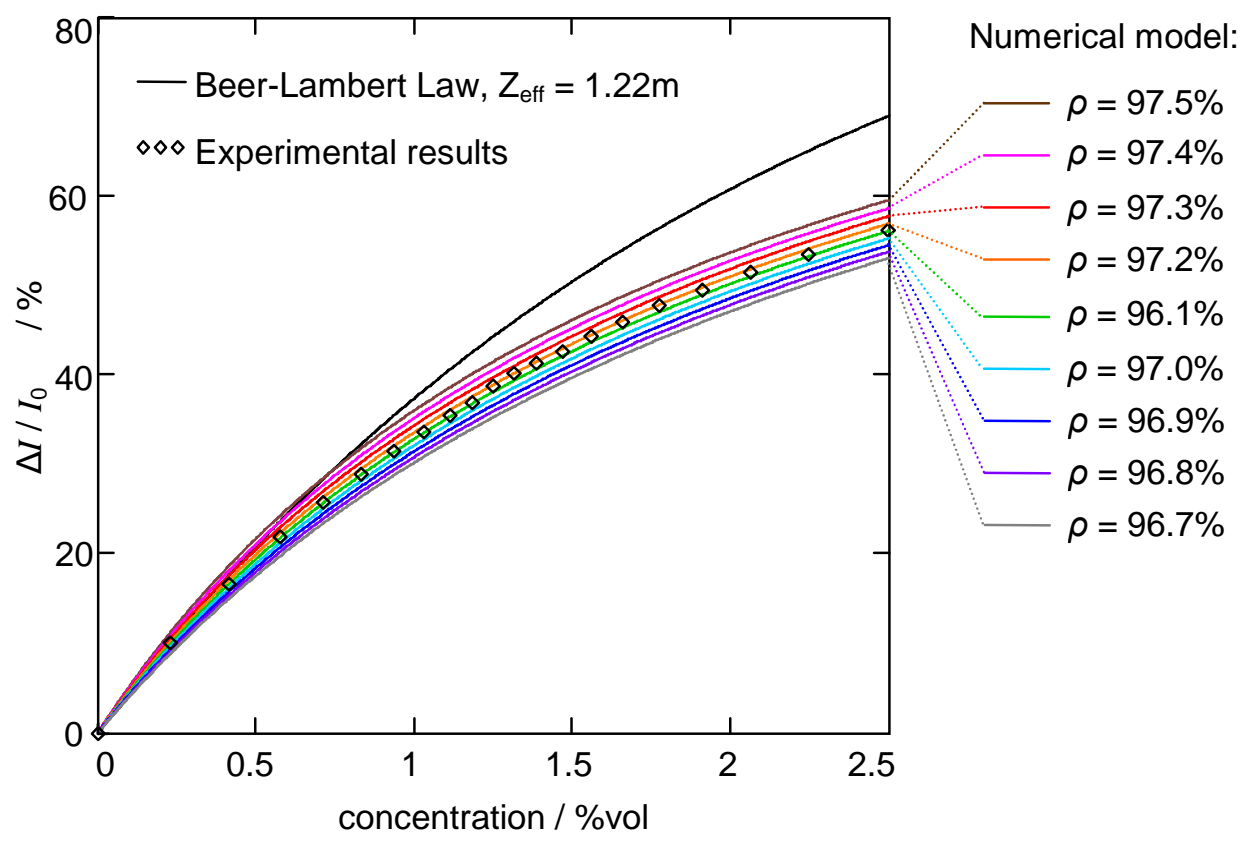

Figure 10. Comparison between experimentally determined response and the results of a full numerical model for a 2 inch integrating sphere with a range of average internal reflectivities. The response predicted by the Beer-Lambert law for a single path-length cell is also provided as a comparison.

Residual errors were calculated as before. We found that errors were minimised for a value of $\rho$ of $97.15 \%$, which is within the estimated error for our calibration value of $97.11 \%$. Errors for three different values of $\rho$, including this optimum value, are plotted against concentration in Figure 11. Again, the results show a consistent systematic pattern that implies that errors in our experimental results may be contributing significantly. Agreement between the model and our experimental data is good, within our estimated error. 


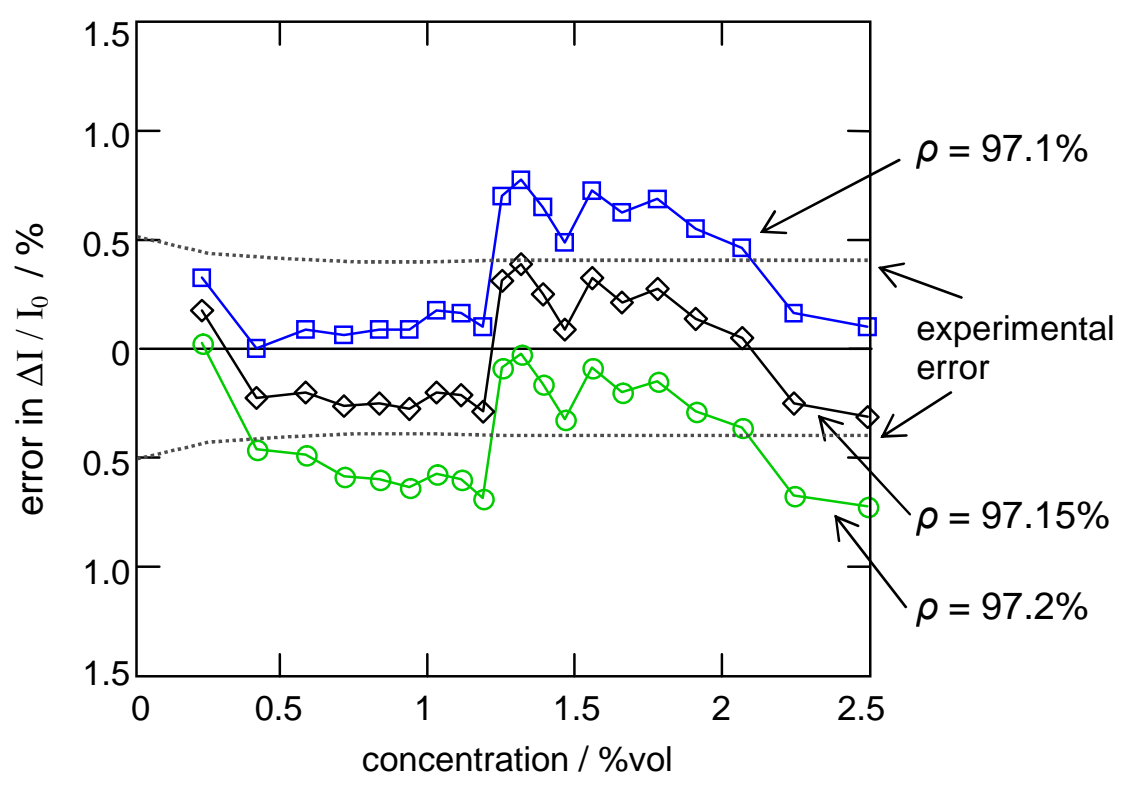

Figure 11. Residual error in response of the system predicted by the full numerical model (as $\left.\Delta \mathrm{I} / \mathrm{I}_{0}\right)$ compared to experimental results, for three modeled values of $\rho$ including the optimum value of $\rho=$ $97.15 \%$.

Figure 12 shows the results of our sensitivity analysis, in which the root-mean-square (rms) residual error was calculated for models using a range of values of $\rho$ lying either side of our calibrated value. As might be expected, the error is highly sensitive to small changes in $\rho$, which implies that for accurate use of integrating spheres as gas cells, cell calibration may be important. Also shown for completeness is the variation in mean effective path length $Z_{\text {eff }}$ with $\rho$. 


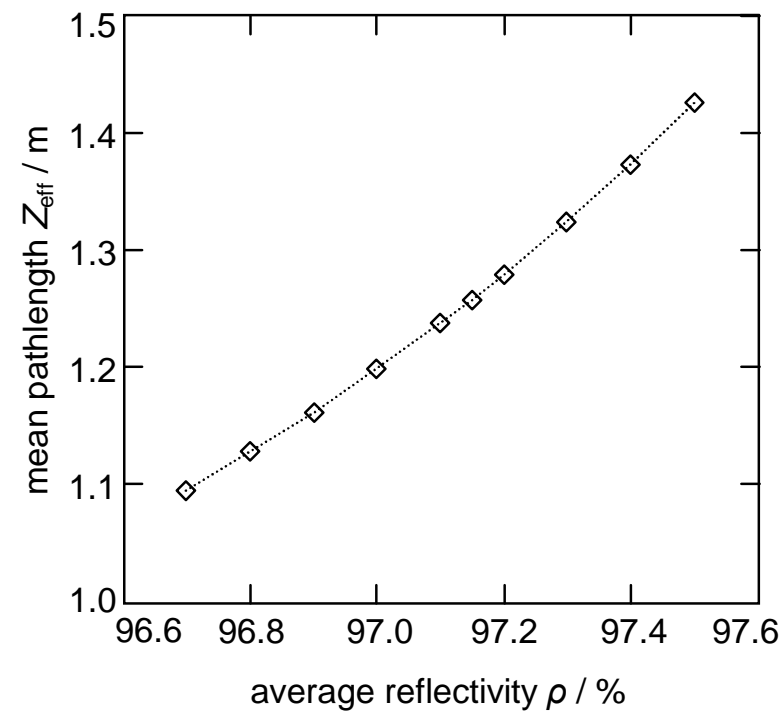

(a)

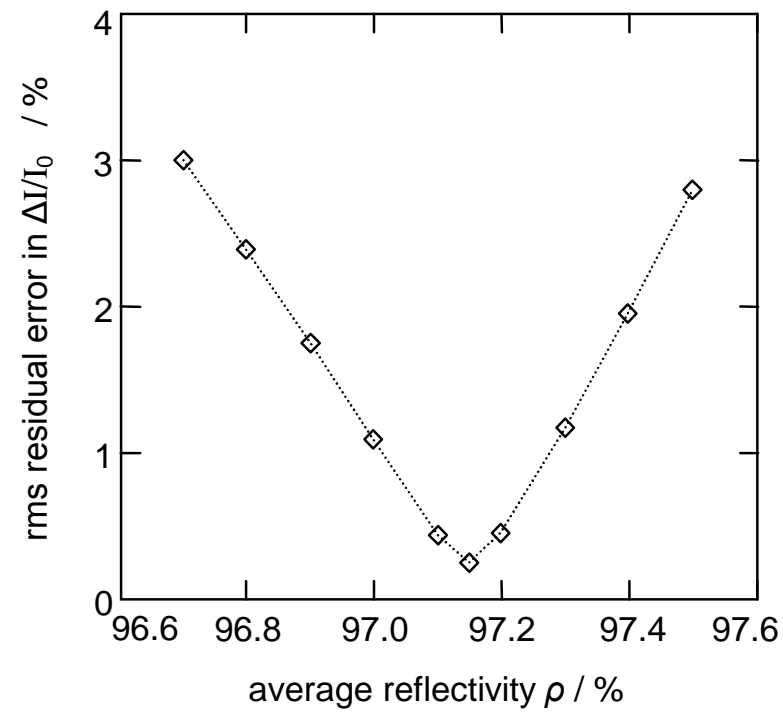

(b)

Figure 12. Sensitivity analysis for modeled response of a $50.8 \mathrm{~mm}$ integrating sphere, showing the variation of (a) $Z_{\text {eff }}$ (the mean effective path length for zero absorption) and (b) rms residual error with the modeled reflectivity $\rho$.

\section{E Summary of residual errors}

Table 2 shows the rms (root mean square) residual errors for comparison between different sets of experimental and modeled data over the concentration range $0-2.5 \%$ volume. Agreement between each of the two models and our experimental data is generally good. Our calibration values of $\rho=97.11 \pm 0.1 \%$ and $\mathrm{z}_{\ell}=2.52 \mathrm{R}$ can be considered a good starting point; the experimental error on $\rho$ was relatively high because this value was based on a measurement at the lowest test concentration. For each model, optimum values of $\rho$ were found, with the new values of $\rho$ lying within $1 \sigma$ of the calibration value. An optimum $z_{\ell}$ was also determined for Eq. (12). Both models agreed well with our experimental data, within our estimated experimental error, therefore we cannot distinguish between them. 
Table 2. Levels of residual error for comparisons between modeled and experimental data.

\begin{tabular}{|l|l|c|c|c|c|}
\hline$\underline{\text { Evaluated model }}$ & $\underline{\text { Condition }}$ & $\underline{\rho} / \%$ & $\underline{\mathrm{Z}}_{\ell}$ & $\frac{\underline{\text { Mean path }}}{\underline{\text { length } \mathrm{Z}_{\text {eff }}}}$ & $\frac{\underline{\text { rms residual error }}}{\underline{\underline{\mathrm{as}} \Delta \mathrm{I} / \mathrm{I}_{0} / \%}}$ \\
\hline Full numerical model & calibration & 97.11 & $2.52 \mathrm{R}$ & 1.242 & 0.37 \\
\hline Eq. (12) & calibration & 97.11 & $2.52 \mathrm{R}$ & 1.253 & 0.43 \\
\hline Full numerical model & optimized & 97.15 & $2.52 \mathrm{R}$ & 1.258 & 0.24 \\
\hline Eq. (12) & optimized & 97.13 & $2.85 \mathrm{R}$ & 1.252 & 0.24 \\
\hline Estimated experimental error & & & 0.4 \\
\hline
\end{tabular}

We can therefore conclude that the simplified model gives a good approximation to the level of light transmission for the $50.8 \mathrm{~mm}$ diameter sphere when used as a gas cell in the range $0-2.5 \%$ vol methane (absorption coefficients of $0-0.01 \mathrm{~cm}^{-1}$ ). The assumption used in this model - that each pass across the cell may be modeled by using the mean path length for a single pass - appears to be a valid one. We suppose that this applies for cells in which the surface has a sufficiently high reflectivity to enable multiple passes to occur, allowing the true path-length distribution to reach its asymptote corresponding to the simplified model (as shown in Figure 9(b)) with low error.

As the results are sensitive to changes in $\rho$, it is perhaps not surprising that, given noise in our data, we should find slightly different optimal values of $\rho$ and $\mathrm{z}_{\ell}$ for the two models. As can be seen from Figure 9, after the first few passes the PDF of path lengths converges to an exponential function as approximated in the simplified model. 


\section{Discussion}

We can use Eq. (12) to predict the response of the sphere at higher concentrations than were used experimentally. Figure 13 shows the response of the sphere extrapolated to a methane concentration of 100 vol.\%, compared with the equivalent response for a conventional cell whose single path length is equal to the value of $Z_{\text {eff }}(1.252 \mathrm{~m})$ calculated for the optimized model (see Table 2).

It can be seen that the response of a single path-length cell quickly becomes saturated as the concentration increases, whereas the integrating sphere's nonlinearity ensures that this saturation occurs at higher concentrations. Whether or not a gas cell is useable at high concentrations depends on the degree to which small differences in absorption may be detected as $\Delta I / I_{0}$. If we assume, for the sake of argument, that a change of 1 part in $10^{3}$ may be just distinguished, measurements made using the single path cell are unable to distinguish between methane concentrations between 14 vol. $\%$ and 100 vol. $\%$. For measurements made using the integrating sphere, the same change in $\Delta I / I_{0}$ corresponds to a change in concentration from 80 vol.\% to 100 vol.\%. Therefore, the dynamic range of the integrating sphere is improved by virtue of its additional nonlinearity. 


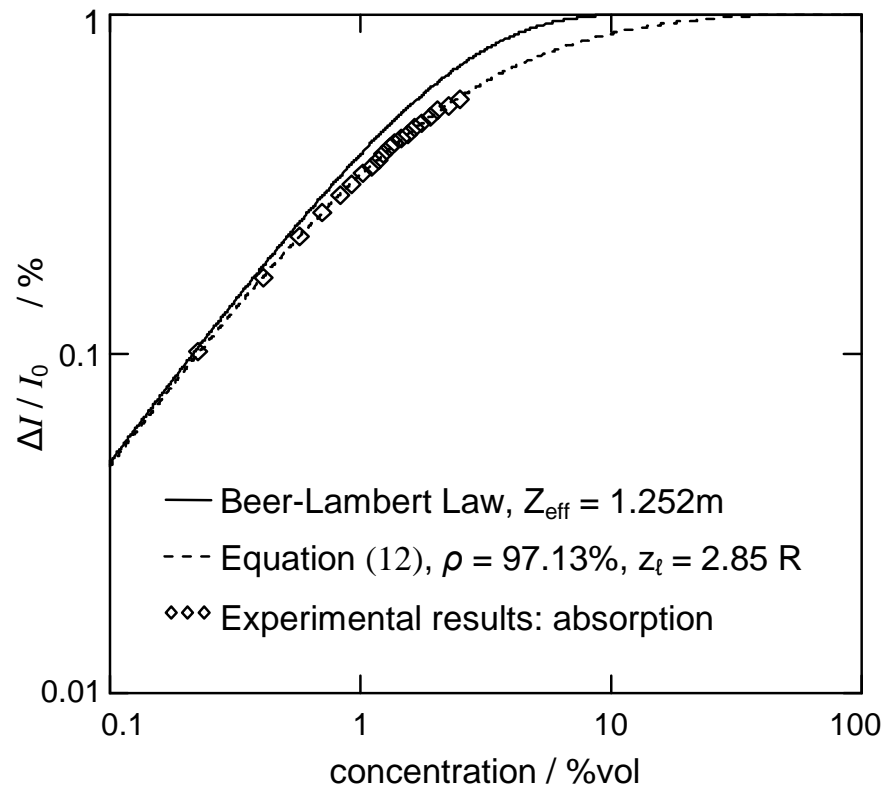

Figure 13. Extrapolated response of integrating sphere to methane concentrations in the range 0.1 to $100 \%$ vol, compared to the response predicted by the Beer-Lambert law for the single path length given in Table 2 for the optimised model.

We can also use our path-length distribution to shows the decay in intensity as a function of time, by scaling from distance $z$ to time in $\mathrm{ct}$, where $\mathrm{c}$ is the speed of light in the medium occupying the sphere and $\mathrm{t}$ is the transit time of a photon. Our results for path-length distributions can therefore be directly related to those of Fry et al. ${ }^{[17]}$ for the temporal decay time of a short pulse in an empty integrating sphere. It is generally accepted that the temporal response shows an exponential decay of the form $I=I_{0} \exp (-\mathrm{t} / \tau)^{[1]}$. However, experimental tests of this are rare. For an ideal intensity impulse, our mean modeled path length should be equivalent to the exponential decay constant.

Fry et al. assumed in their model that the transit distance for a single pass across the sphere is equal to the mean path length; we continued to use this approach in the simplified model considered here. For their case (ii), they considered zero time to be at the time of light entry to the cell, with a first pass across the cell of 
length equal to $\frac{4}{3} R$ (ie modelling an ideally diverging source). We altered our model to take account of this (removing our launch and de-launch path $z_{\ell}$ ) and ran the calculation for their conditions of a sphere with radius $\mathrm{R}$ and an internal reflectivity $\rho$ of $99.0 \%$. Because the mean reflectivity was higher than for our previous work, we ran the numerical model for a greater number of iterations (1000) to enable the model to converge (in other words, the modeled path lengths were longer in this case). The results in Table 3 are expressed in the same terms used by Fry et al., the dimensionless quantity c $\tau / \mathrm{R}$ (equivalent in our model to $\left.\mathrm{Z}_{\text {eff }} / \mathrm{R}\right)$, and show good agreement between the models. Agreement between our simplified analytical model and theirs is guaranteed by Eq. (9), which derives from their work.

Table 3. Comparison of the results of our model with the previous theoretical work of Fry et al. ${ }^{[17]}$.

\begin{tabular}{|l|l|c|}
\hline \multirow{2}{*}{ Study } & \multicolumn{1}{|l|}{ Model and parameters } & Exponential decay time, $c \tau / R$ \\
\hline \multirow{2}{*}{ Fry et al. ${ }^{[17]}$} & Analytical derivation, case (ii), $\rho=99.0 \%$ & 132.7 \\
\cline { 2 - 3 } & Monte Carlo simulation, case (ii), $\rho=99.0 \%$ & 134.0 \\
\hline \multirow{2}{*}{ This paper } & Full model, $\rho=99.0 \%$ & 134.0 \\
\cline { 2 - 3 } & $\begin{array}{l}\text { Simplified model, } \rho=99.0 \% \\
\text { (see Eq. (12)) }\end{array}$ & $\delta=132.7$ \\
& & $\delta+\frac{z_{0}}{2}=133.4$ \\
\hline
\end{tabular}

It is interesting that the result of our numerical model agrees so well with that of Fry et al.'s Monte Carlo approach, yet the results of both numerical models differ from those of the analytical models. For our simple analytical model, following Eq. (12) and Table 1, we find that the use of our additional offset of $z_{0} / 2$ improves agreement with the numerical models but does not fully account for the difference. 
Fry et al. did not provide an experimental test of their model, presumably because of the practical difficulty associated with measuring pulse decay times with sub-nanosecond resolution. Our experimental results therefore provide an indirect though possibly simpler test of both our model and theirs.

Integrating spheres of are great utility in measurement applications that require an even and wellcharacterized irradiance across the whole internal surface. To use such a sphere as a gas absorption cell, our requirements are less stringent and may be listed as follows:

(i) For high sensitivity, we require a long path length contained within a practical sampling volume.

(ii) For tolerance to misalignment during manufacture and / or vibration in use, we require the irradiance be even across the surface in a region local to the detector, but not more widely.

(iii) For ease of calibration by manufacturers it is preferred to have repeatable path lengths, requiring both repeatable surface reflectivities and repeatable cell geometries.

For an integrating cavity the ideal solution is a sphere, but this is not an absolute requirement. The fact that our model is not very sensitive to the path-length distribution for a single pass indicates that other cavity geometries may give similar results if they maintain the same mean path length. Other geometries might be easier to manufacture repeatably and at low cost. This was also considered in the work of Fry et al. ${ }^{[17]}$.

Instrument users typically prefer outputs that are linear with the target measurand. At high analyte concentrations, absorption measurements based on the use of the Beer-Lambert law will always have to contend with some nonlinearity, which is compounded when path-length distributions are broad. However, as long as nonlinearities are predictable, manufacturers are able to linearise the output using algorithms or look-up tables. 


\section{Conclusion}

We have developed a model that describes the variation in transmission with analyte concentration for an integrating sphere used as an absorption cell. This model has been tested by measuring the absorption of gaseous methane at $1651 \mathrm{~nm}$ in the concentration range $0-2.5$ vol.\% (corresponding to an absorption range

of $\left.0-0.01 \mathrm{~cm}^{-1}\right)$. Within the model, the probability density function (PDF) of optical path lengths within the sphere is calculated, and the fractional light transmission determined using the Beer-Lambert Law for light within each path-length interval in the PDF. The total light transmission shows an additional nonlinearity beyond what would normally be expected from the Beer-Lambert law for a gas cell with a single path length, and this nonlinearity is shown to be in good agreement with our experimental data.

Because the full model is computationally intensive, we have also tested a simplified model, whereby each

pass across the integrating sphere is assigned a length equal to the average length pass across the cell $\left(\frac{4}{3} R\right)$. This yields a simple analytical relationship that can be easily fitted to experimental data by varying two parameters, the average sphere reflectivity $\rho$ and the launch path length $z_{\ell}$, in our case yielding good agreement with residual errors within our estimated experimental error. This simpler model is a development of previous work in the field, and our experimental data provides a validation for its use.

\section{Acknowledgements}

This work has been supported by the Engineering and Physical Sciences Research Council (EPSRC), UK under Grant GR/T04601/01. J. Hodgkinson is supported by an EPSRC Advanced Research Fellowship, GR/T04595/01. 


\section{References}

[1] Labsphere Inc. "A guide to integrating sphere theory and applications," Labsphere, North Sutton, NH, USA (1998).

[2] P. Elterman, "Integrating cavity spectroscopy,” Appl. Opt. 9 (9), 2140-2142 (1970)

[3] E.S. Fry, G.W. Kattawar and R.M. Pope, "Integrating cavity absorption meter," Appl. Opt. 31 (12), 2055-2065 (1992)

[4] I. Fecht and M. Johnson, "Non-contact, scattering-independent water absorption measurement using a falling stream and integrating sphere,” Meas. Sci. Technol. 10 612-618 (1999)

[5] J. Hodgkinson, M. Johnson and J.P. Dakin, "Performance of a photothermal detector with turbid liquids,” Appl. Opt. 44, 4360-4367 (2005).

[6] C.G. Venkatesh, R.S. Eng and A.W. Mantz, “Tunable diode laser integrating sphere systems: a study of their output intensity characteristics,” Appl. Opt. 19 (10) 1704-1710 (1980).

[7] R.M. Abdullin and A.V. Lebedev, "Use of an integrating sphere as a multipass optical cell," Sov. J. Opt. Technol. 55 (3) 139-41 (1988).

[8] S. Tranchart, I.H. Bachir and J.-L. Destombes, "Sensitive trace gas detection with near-infrared laser diodes and an integrating sphere," Appl. Opt. 35 (36), 7070-7074 (1996).

[9] J.U. White, "Long Optical Paths of Large Aperture," Journal of the Optical Society of America 32, 285. (1942)

[10] D.R. Herriott, H. Kogelnik and R. Kompfner, "Off-Axis Paths in Spherical Mirror Interferometers," Appl. Opt. 3, 523 (1964)

[11] S.M. Chernin and E.G. Barskaya, "Optical multipass matrix system,” Appl. Opt. 30 (1), $51-58$ (1991)

[12] E. Hawe, P Chambers, C. Fitzpatrick and E. Lewis, " $\mathrm{CO}_{2}$ monitoring and detection using an integrating sphere as a multipass absorption cell,” Meas. Sci. Technol. 18, 3187-3194 (2007)

[13] E. Hawe, C. Fitzpatrick, P. Chambers, G. Dooly and E. Lewis, "Hazardous gas detection using an 
integrating sphere as a multipass gas cell,” Sensor. Actuat. A 141, 414-421 (2008)

[14] S.C. Cutler and A. Vass, “Gas Sensor,” International patent, publication number WO 2005 / 054827 A1 (2005)

[15] The Institution of Gas Engineers and Managers, Dealing with reported gas escapes, Safety Recommendations IGEM/SR/20 Edition 2. (IGEM, London 1998).

[16] J.T.O. Kirk, "Modeling the performance of an integrating cavity absorption meter: theory and calculations for a spherical cavity,” Appl. Opt. 34 (21), 4397-4408 (1995).

[17] E.S.Fry, J. Musser, G.W. Kattawar and P.-W. Zhai, "Integrating cavities: temporal response,” Appl. Opt. 45 (36), 9053-9065 (2006).

[18] J.D. Ingle and S.R Crouch, Spectrochemical analysis. (Prentice Hall, New Jersey, 1988)

[19] L.S. Rothman, D. Jacquemart, A. Barbe, D.C. Benner, M. Birk, L.R. Brown, M.R. Carleer, C. Chackerian, Jr., K. Chance, L.H. Coudert, V. Dana, V.M. Devi, J.-M. Flaud, R.R. Gamache, A. Goldman, J.-M. Hartmann, K.W. Jucks, A.G. Maki, J.-Y. Mandin, S.T. Massie, J. Orphal, A. Perrin, C.P. Rinsland, M.A.H. Smith, J. Tennyson, R.N. Tolchenov, R.A. Toth, J. VanderAuwera, P. Varanasi, and G.Wagner, “The HITRAN 2004 molecular spectroscopic database,” J. Quant. Spectrosc. Ra. 96, 139-204 (2005). 